\title{
PRESERFLO MicroShunt
}

\author{
Nathan M. Kerr, lqbal Ike K. Ahmed, Leonard Pinchuk, \\ Omar Sadruddin, and Paul F. Palmberg
}

\subsection{Introduction}

Reducing intraocular pressure (IOP) remains the only proven treatment to prevent vision loss from glaucoma [1-3]. Under-treatment of glaucoma remains a significant issue and inadequate IOP reduction can increase the risk of vision loss [4]. Traditionally, trabeculectomy has been regarded as the gold standard in glaucoma surgery since it was described in the 1960s [5]. However, while one of the most effective IOP-lowering treatments, severe adverse events can occur, recovery can be prolonged and intense post-operative management is required.

\footnotetext{
N. M. Kerr $(\bowtie)$

Centre for Eye Research Australia, Melbourne, VIC, Australia

Royal Victorian Eye and Ear Hospital, Melbourne, VIC, Australia

e-mail: nathan.kerr@eyeandear.org.au
}

\section{I. K. Ahmed}

Department of Ophthalmology and Vision Sciences, University of Toronto,

Toronto, ON, Canada

Prism Eye Institute, Mississauga, ON, Canada

L. Pinchuk

Ophthalmic Biophysics Center, Bascom Palmer Eye Institute, University of Miami Miller

School of Medicine, Miami, FL, USA

Santen Inc., Emeryville, CA, USA

O. Sadruddin

Santen Inc., Emeryville, CA, USA

P. F. Palmberg

Bascom Palmer Eye Institute, University of Miami Miller School of Medicine,

Miami, FL, USA

Anne Bates Leach Eye Hospital, Miami, FL, USA 
Trabeculectomy is well-recognized to achieve significant and sustained reductions in IOP. In the tube versus trabeculectomy (TVT) study the mean IOP following trabeculectomy was $12.6 \mathrm{mmHg}$ at 5 years. However, $37 \%$ of patients in this study developed complications in the early perioperative period and $18 \%$ required re-operation [6]. Furthermore, there is a significant surgical learning curve associated with trabeculectomy and a high level of surgical skill is required [7]. Intensive postoperative follow-up is mandatory to achieve optimum outcomes and perioperative interventions are frequent, with up to $78 \%$ of cases requiring some form of bleb manipulation and/ or suture removal [8]. Although rare, sight-threatening complications following trabeculectomy can occur and include hypotony, choroidal effusions and suprachoroidal haemorrhage among others [6]. For these reasons, trabeculectomy is often reserved for late in the disease process when other treatments have failed [9].

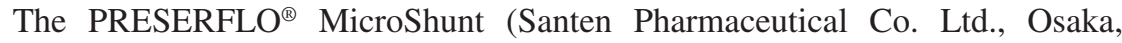
Japan) is a new glaucoma drainage microtube that has been developed with the intention to provide significant and long-term IOP and glaucoma medication reduction similar to that of trabeculectomy but in a simpler, safer and less-invasive operation with faster recovery than conventional surgery. This chapter summarizes the development, lab testing, pre-clinical studies and human clinical trials for the MicroShunt.

\subsection{Device Design and Development}

There have been three major iterations in the design of the shunt to enhance ease of insertion, minimize complications and improve success rates. The final design, the PRESERFLO MicroShunt, is $8.5 \mathrm{~mm}$ long with an internal lumen of $70 \mu \mathrm{m}$ and an outer diameter of $350 \mu \mathrm{m}$ (Fig. 7.1). It received a CE mark in 2012. The length of the device was designed to achieve $1-2 \mathrm{~mm}$ in the anterior chamber, $3 \mathrm{~mm}$ within the sclera and $3 \mathrm{~mm}$ posterior to the needle tract wound. These dimensions summed to $8.5 \mathrm{~mm}$ in length. The lumen diameter was then approximated with the HagenPoiseuille equation to provide sufficient resistance to limit hypotony given published aqueous humour flow rates and viscosities [11]. The shunt is made of poly(styrene-block-isobutylene-block-styrene) or 'SIBS' material. The anterior end is bevelled to facilitate entry into the scleral tunnel and anterior chamber and is

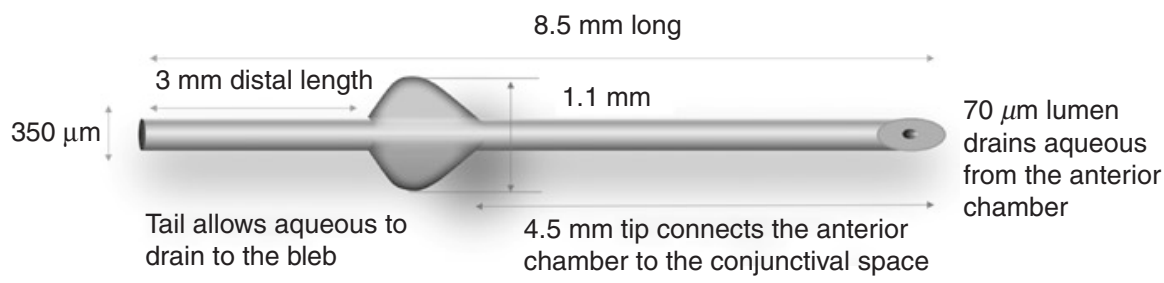

Fig. 7.1 PRESERFLO ${ }^{\circledR}$ MicroShunt. (Copyright Santen Pharmaceutical Co. Ltd., Osaka, Japan; reproduced with permission) 
faced towards the cornea to enable visualization and clearing of the lumen if obstructed. Fixation fins are located approximately half-way down the shunt and serve three important functions. The fins fit snugly within the scleral tunnel and are designed to prevent leakage of aqueous around the tube, stop migration of the tube into the anterior chamber and orientate the shunt so that the bevel faces the cornea. The final design does not have a plate, reducing the complexity of insertion and minimizing the risk of diplopia.

The initial design, called the MIDI-Tube (an acronym for Miami InnFocus Drainage Implant), was trimmed in situ by the surgeon to approximately $11 \mathrm{~mm}$ long with an internal lumen of $70 \mu \mathrm{m}$ and an outer diameter of $250 \mu \mathrm{m}$ (Fig. 7.2). Early animal studies assessed the effect of lumen size on IOP lowering and found fewer complications and comparable IOP levels with a $70 \mu \mathrm{m}$ lumen compared to larger $100 \mu \mathrm{m}$ and $150 \mu \mathrm{m}$ lumens [10]. A single fixation tab was located on one side to prevent migration into the anterior chamber and to enable insertion through a slotted needle with the fin protruding through the slot. The device was inserted through the scleral tunnel using this slotted needle inserter, however, due to the soft and flexible characteristics of the tube it would frequently jam in the inserter, and the surgeon would instead simply thread the MicroShunt through the scleral tunnel with forceps. The initial success rate, defined as an IOP $\leq 21 \mathrm{mmHg}$ with a reduction from baseline of $\geq 20 \%$, was low at $42 \%$ at 1 year in early human clinical trials [11]. However, it should be noted that in the first human trial (Bordeaux I), antimetabolites were not used and more than half of the patient population had failed previous glaucoma surgeries. In a subsequent clinical trial of 16 patients with previous failed incisional surgery (Bordeaux II), mitomycin-C (MMC) $0.2 \mathrm{mg} / \mathrm{mL}$ was applied for 2-3 min to only the sclera in the vicinity of the tube, and the success rate increased to $67 \%$ at 1 year [11].

A second-generation device, called the MIDI-Ray as it resembled a sting ray, was developed comprising a larger tube measuring $12 \mathrm{~mm}$ in length with an outer diameter of $350 \mu \mathrm{m}$, internal lumen of $100 \mu \mathrm{m}$ connected to a plate $7 \mathrm{~mm}$ in diameter (Fig. 7.2) [11]. It was hypothesized that the plate would prevent encapsulation and eliminate the need for anti-metabolites [11]. However, the device had a high rate of hypotony due to the larger diameter tube and undesirable cystic bleb morphology. The MIDI-Ray did not enter large scale clinical trials.
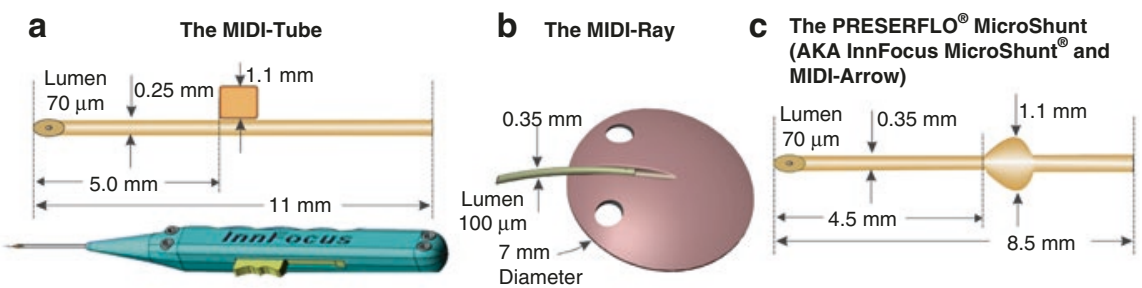

Fig. 7.2 The three major iterations in the design of the glaucoma shunt: (a) the MIDI-Tube; (b) the MIDI-Ray and (c) the PRESERFLO ${ }^{\circledR}$ MicroShunt, which is the final design. (Copyright Santen Pharmaceutical Co. Ltd., Osaka, Japan; reproduced with permission) 
Fig. 7.3 Anterior segment photograph showing (a) the PRESERFLO $^{\circledR}$ MicroShunt in the anterior chamber and (b) a diffuse bleb associated with the PRESERFLO ${ }^{\circledR}$ MicroShunt. (Copyright Chelvin Sng, FRCSEd; reproduced with permission)
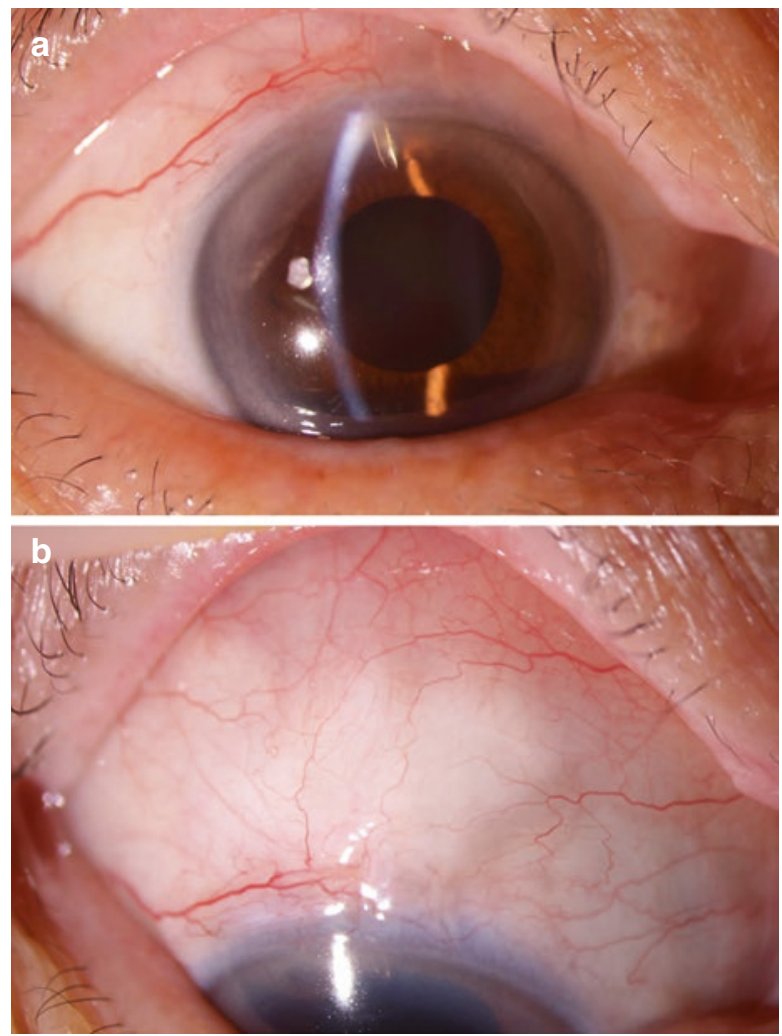

The third-generation device, called the MIDI-Arrow, comprised of symmetrical planar fins with a wingspan of $1.1 \mathrm{~mm}$ located midway along the length of an $8.5 \mathrm{~mm}$ SIBS tube with a $350 \mu \mathrm{m}$ outer diameter and a $70 \mu \mathrm{m}$ lumen. The MIDIArrow name was dropped due to concerns about patients believing an arrow would be placed in their eye. At that time, many of the implanting surgeons simply called the device the 'InnFocus device' or the 'InnFocus procedure', therefore the device was called the InnFocus MicroShunt ${ }^{\circledR}$, often referred to as simply the 'IMS'. Santen Pharmaceutical Co. Ltd. acquired InnFocus Inc. in May 2016 and 3 years later renamed the device the PRESERFLO MicroShunt (Fig. 7.3).

\subsection{Development of SIBS}

Inflammation and fibrosis in the subconjunctival space may lead to surgical failure. Therefore, the selection of a biocompatible material that produces minimal inflammation is important to increase success. Traditional glaucoma drainage devices such as the Molteno (Molteno Ophthalmic Limited, Dunedin, New Zealand) or Baerveldt (Abbott Medical Optics, Santa Ana, CA) have been shown to elicit intense 
inflammatory reactions with multinucleated giant cells and the deposition of dense fibrous tissue surrounding the implants $[12,13]$.

Conventional implant materials such as polyether urethane slowly degrade in the body due to hydrolysis and oxidation [14]. This degradation attracts granulocytes (e.g. polymorphonuclear leukocytes) and macrophages leading to a foreign body reaction and capsule formation; features undesirable for a subconjunctival filtration device [15]. Silicones are more biostable than the polyurethanes but are often contaminated with unreacted starting materials as well as fillers and elicit a foreign body reaction [14]. The PRESERFLO MicroShunt is made of ultrapurified medicalgrade poly(styrene-block-isobutylene-block-styrene) or SIBS, a novel biostable and biocompatible synthetic polymer devoid of cleavable group such as amides, esters, ureas, carbamates, etc. on both its backbone and side groups. First approved for medical use in 2004, SIBS has been widely used in cardiac stents due to its lack of biodegradation, absence of platelet activation, minimal tissue reaction and ability to be used as a drug-eluting system $[16,17]$.

Poly(styrene-block-isobutylene-block-styrene) is a synthetic polymer devoid of any sites prone to degradation by mechanisms such as oxidation, hydrolysis or enzymatic degradation (Fig. 7.4) [16]. Consisting of stable alternating secondaryand-quaternary carbons, SIBS is not prone to double bond formation that could lead to embrittlement or stress cracking [16]. Synthesized by cationic polymerization, SIBS can be injection and compression moulded and is stable under harsh conditions [18]. The SIBS material is soft and flexible and will conform to various shapes without surface cracking [19]. In addition, SIBS is a thermoformable material and will assume the shape it is placed in without a tendency to straighten as do the silicones tubes which are thermoset materials that tend to straighten over time. Silicones need to be secured in place, usually covered with a patch graft, in contrast to SIBS tubes which assume the shape of the globe. SIBS is sterilizable with ethylene oxide but not gamma-ray irradiation [19].

The biocompatibility of SIBS has been established in both animal and human studies. Discs of SIBS material were implanted in the cornea and sub-Tenon space

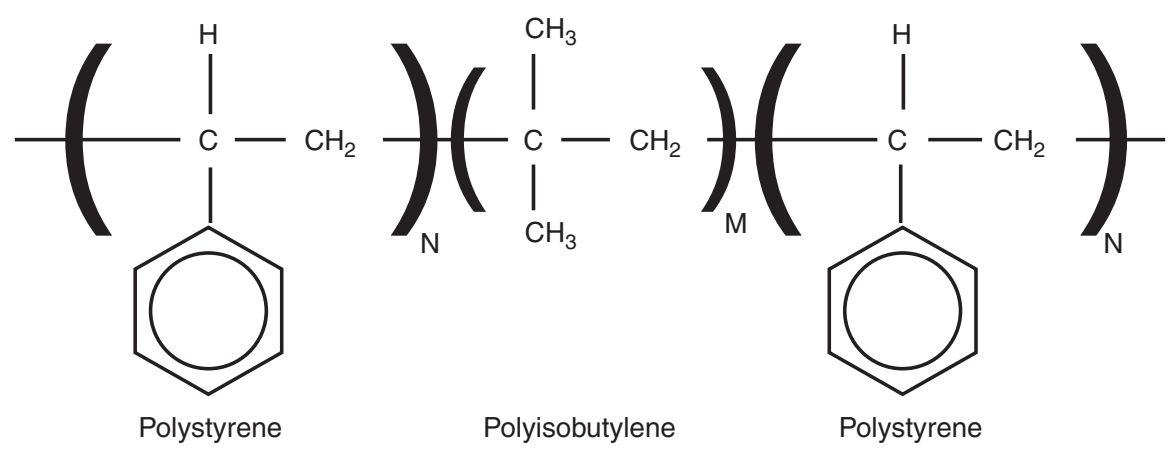

Fig. 7.4 Simplified structure of poly(styrene-block-isobutylene-block-styrene), or 'SIBS'. (Copy right Santen Pharmaceutical Co. Ltd., Osaka, Japan; reproduced with permission) 
of normal rabbit eyes and compared against discs of silicone rubber [20]. The silicone discs showed angiogenesis, myofibroblasts and capsule formations, whereas there was no angiogenesis, myofibroblasts, nor intact capsules surrounding the SIBS material [20]. Further studies investigated the biocompatibility of a glaucoma drainage device made from SIBS in normal New Zealand white rabbits [21]. Glaucoma drainage devices made of SIBS were implanted into the anterior chamber while the distal tip was placed in the subconjunctival space [21]. Control animals underwent insertion of a similarly designed silicone tube [21]. At 3 months, haematoxylin-eosin staining revealed abundant collagen IV deposition around the silicone tubes with expression of alpha-smooth muscle actin [21]. In comparison, eyes implanted with the SIBS device showed a distinct absence of myofibroblasts, inflammatory cells and fibrosis [21]. In addition, encapsulation and neovascularisation were not observed in the SIBS group.

Glaucoma device patency was assessed by injection of fluorescein into the anterior chamber. All SIBS tubes remained patent out to 6 months while only two of the six silicone tubes were patent at 3 months [21].

\subsection{Clinical Data}

The safety and efficacy of the PRESERFLO MicroShunt have been evaluated in a number of clinical trials. In a multicentre, retrospective clinical trial, Beckers et al. reported the results of 91 patients with open-angle glaucoma who underwent either stand-alone MicroShunt insertion $(n=73)$ or combined cataract surgery and MicroShunt insertion $(n=18)$ [22]. All cases received MMC, but a varying concentration $(0.2-0.4 \mathrm{mg} / \mathrm{mL}$ on sponges for $2-3 \mathrm{~min})$. At 12 months, the mean IOP had reduced from a baseline of $24.3 \mathrm{mmHg}$ on 2.4 medications to $13.3 \mathrm{mmHg}$ on 0.4 medications with $83 \%$ of patients off all glaucoma medications [22]. The most common adverse event was transient numerical hypotony, occurring in $11 \%$ of patients [22]. All cases of hypotony resolved without intervention [22].

A second clinical trial examined the effect of MMC concentration on surgical outcomes [23]. In this retrospective two-centre study, patients with open-angle glaucoma were implanted with either the MicroShunt alone $(n=66)$ or in combination with cataract surgery $(n=21)$ [24]. Patients received either MMC $0.2 \mathrm{mg} / \mathrm{mL}$ near the limbus, MMC $0.4 \mathrm{mg} / \mathrm{mL}$ near the limbus or MMC $0.4 \mathrm{mg} / \mathrm{mL}$ deep in the subconjunctival conjunctival space [23]. For all patients, the duration of MMC exposure was 2-3 min. Overall there was a reduction in IOP and glaucoma medication requirements in all groups with the lowest IOP being in patients treated with MMC $0.4 \mathrm{mg} / \mathrm{mL}$ near the limbus [23]. In these patients, IOP reduced from a mean of $23.8 \pm 5.3 \mathrm{mmHg}$ on $2.4 \pm 0.9$ medications at baseline to $10.7 \pm 2.8 \mathrm{mmHg}$ on $0.3 \pm 0.8$ medications at 12 months [23]. There were no sight-threatening events in any group [23].

The patients who received MMC $0.4 \mathrm{mg} / \mathrm{mL}$ near the limbus were followed prospectively and the results presented at 2 and 3 years [16, 24]. All patients had 
primary open-angle glaucoma and had failed maximum tolerated medical therapy [16]. Patients with failed subconjunctival filtration surgery were excluded [16]. From a baseline IOP of $23.8 \pm 5.3 \mathrm{mmHg}$ on maximum tolerated medication therapy, $100 \%$ of patients achieved an IOP of $18 \mathrm{mmHg}$ or lower at both 1 and 2 years [16]. The most common adverse events were mild and consisted of transient shallow anterior chamber and IOP $<5 \mathrm{mmHg}(3 / 23,13 \%)$ [16].

At 3 years, $100 \%$ of the patients continued to achieve an IOP $\leq 18 \mathrm{mmHg}$ and 18 $(82 \%)$ had an IOP of $\leq 14 \mathrm{mmHg}$ [24]. The mean IOP at 3 years was $10.7 \pm 3.5 \mathrm{mmHg}$, representing a 50\% reduction in IOP [24]. Mean medication usage reduced from a baseline of $2.4 \pm 0.9$ medications to $0.7 \pm 1.1$ and $80 \%$ of patients were off glaucoma medications [24]. There were no sight-threatening complications and no patient lost $>1$ line of visual acuity [24]. The most common adverse events were transient hypotony $(3 / 23,13 \%)$ and transient choroidal effusions $(2 / 23,8.7 \%)$ [24]. All resolved spontaneously [24]. There were no cases of bleb leak, infection, tube exposure or persistent corneal oedema [24].

In August 2019, Santen Pharmaceutical announced the results of the US premarket approval (PMA), head-to-head study of the PRESERFLO MicroShunt versus trabeculectomy [25]. This prospective, randomized, controlled, single-masked, multicentre study compared standalone MicroShunt implantation with intraoperative $0.2 \mathrm{mg} / \mathrm{mL}$ MMC against stand-alone trabeculectomy with the same concentration of MMC. At 12 months, mean ( \pm standard deviation) diurnal IOP was reduced from $21.1 \pm 4.9 \mathrm{mmHg}$ to $14.2 \pm 4.4 \mathrm{mmHg}$ in the MicroShunt group and from $21.1 \pm 5.0 \mathrm{mmHg}$ to $11.2 \pm 4.2 \mathrm{mmHg}$ in the trabeculectomy group. The mean number of glaucoma medications at month 12 was reduced in both groups, from 3.0 medications at baseline to 0.6 in the MicroShunt group and 0.3 in the trabeculectomy group, with $71.6 \%$ of subjects in the MicroShunt group being medication-free compared with $84.8 \%$ of subjects in the trabeculectomy group. Although the 12-month IOP was statistically lower in the trabeculectomy group compared with the MicroShunt group, trabeculectomy was associated with a greater incidence of hypotony at any time (51.1\% vs. $30.6 \%)$, bleb leaks and lens opacity.

\subsection{Patient Selection}

The PRESERFLO MicroShunt is indicated for patients with open-angle glaucoma refractory to medical therapy. The MicroShunt can be performed alone or in combination with cataract surgery for patients with both refractory glaucoma and visually significant cataract. The MicroShunt provides a simple and fast alternative to primary trabeculectomy and eliminates the need for scleral flap dissection, iridectomy and post-operative suture lysis. Once efficacy and safety are fully established, the MicroShunt may allow for an earlier transition to surgical management. Because a significant proportion of patients are medication-free following the MicroShunt procedure compared to other MIGS procedures, it may be especially suited for patients with intolerance to topical glaucoma medications or adherence problems. 
Due to its ability to achieve IOP in the low to mid-teens, the MicroShunt may be appropriate for patients with more advanced disease, unlike some other minimally invasive procedures. The MicroShunt can theoretically be placed in any quadrant, however as with trabeculectomy, prior failed subconjunctival filtration surgery may reduce surgical success rates. Inferior placement may increase the risk of blebrelated infection and is not recommended.

The PRESERFLO MicroShunt may be useful in the treatment of angle-closure glaucoma when combined with cataract surgery or in pseudophakic patients. Because the PRESERFLO MicroShunt shares features of both trabeculectomy and a glaucoma drainage device, it may potentially have a role in conditions such as iridocorneal endothelial cell syndrome, uveitic glaucoma and neovascular glaucoma. However, at the present time, there is limited data on the efficacy and safety of the device for these off-label indications.

\subsection{Surgical Technique}

The PRESERFLO MicroShunt comes in a sterile pre-packaged kit containing a $3 \mathrm{~mm}$ marking ruler, marking pen, sponges to apply MMC, a $1 \times 1 \mathrm{~mm}$ triangular slit keratome knife to create a scleral pocket, and either a $25 \mathrm{G}$ or $27 \mathrm{G}$ needle.

The procedure is most commonly performed under a local anaesthetic block and the preferred site for implantation is superiorly at 11 or 1 o'clock (Fig. 7.5). Initially, a 6- to 8-mm wide peritomy is made at the limbus before Westcott scissors are used to dissect posteriorly for $8-10 \mathrm{~mm}$, ensuring to dissect under Tenon's capsule. Bipolar diathermy is then used to achieve haemostasis before MMC application. Three sponges soaked in MMC, typically between 0.2 and $0.4 \mathrm{mg} / \mathrm{mL}$, are placed under Tenon's for 3 min before irrigation with $>20 \mathrm{~mL}$ of normal saline. Care should be taken to apply the sponges close to the limbus as well as deep in the flap. The

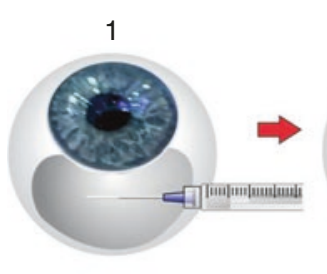

5

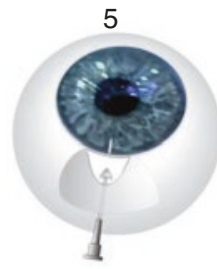

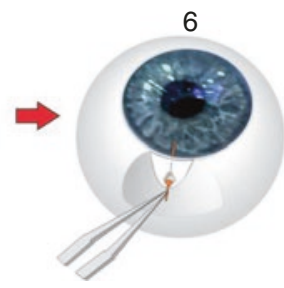

3
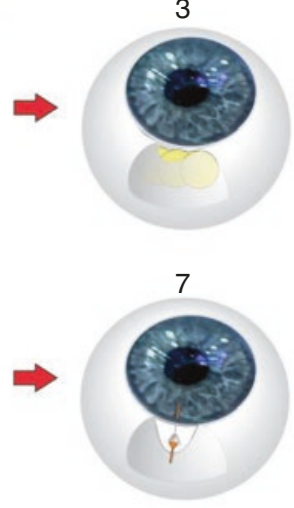

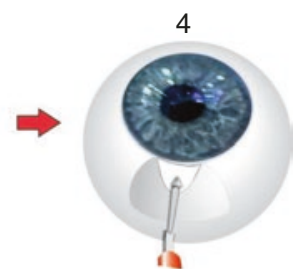

8

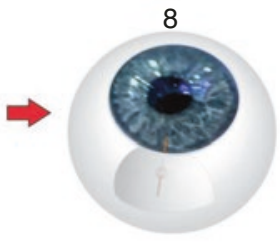

Fig. 7.5 PRESERFLO ${ }^{\circledR}$ MicroShunt implantation. (Copyright Santen Pharmaceutical Co. Ltd., Osaka, Japan; reproduced with permission) 
ruler is used to mark $3 \mathrm{~mm}$ and a scleral tunnel fashioned with a slit keratome for $2 \mathrm{~mm}$. A $25 \mathrm{G}$ needle is passed within the scleral tunnel to the apex before entering the anterior chamber in the plane of the iris and away from the corneal endothelium. The MicroShunt is inserted into the scleral tunnel with forceps and the wings secured within the tunnel (Fig. 7.6). The distal end is observed for flow (Fig. 7.7). If there is no flow, gentle pressure can be applied to the globe or the shunt can be flushed with a thin-walled $23 \mathrm{G}$ cannula if required. The distal end is tucked under Tenon's, which is advanced with the conjunctiva back to the limbus prior to conjunctival closure. The distal end should be checked to ensure it is not occluded with Tenon's and lastly it should be verified that there is no leak.
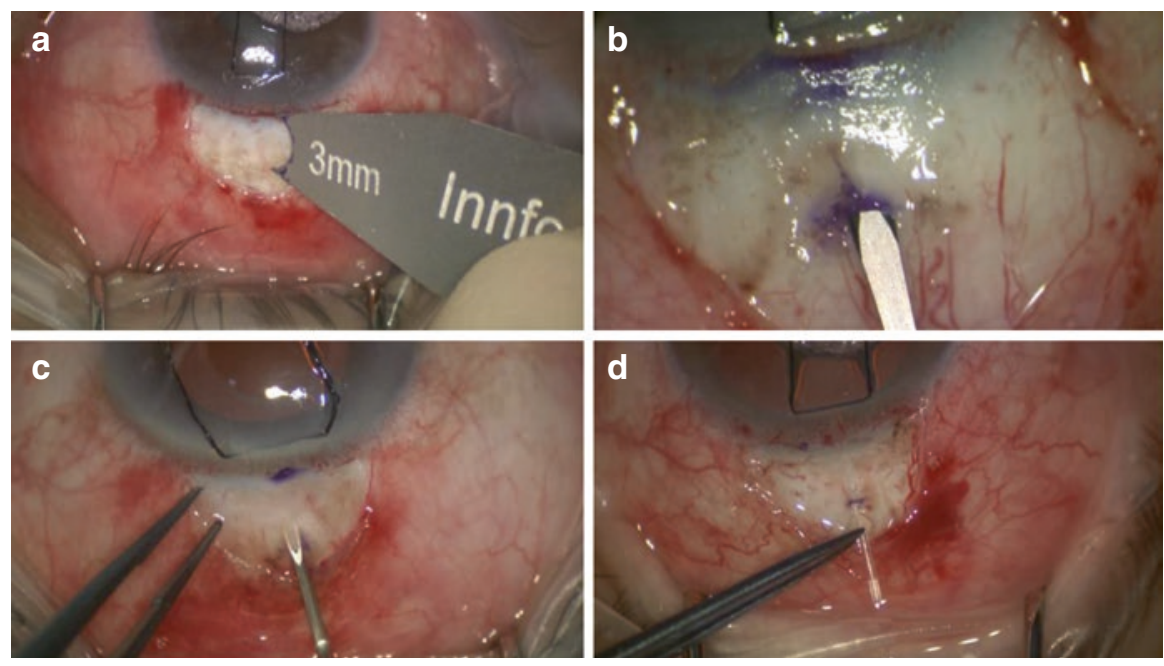

Fig. 7.6 Insertion of the PRESERFLO ${ }^{\circledR}$ MicroShunt. (a) A mark is made $3 \mathrm{~mm}$ from the surgical limbus. (b) A 2-mm shallow tunnel is formed in the sclera using a slit knife. (c) A 25-guage needle is passed through the tunnel to enter the anterior chamber. (d) The MicroShunt is inserted through the needle tract with the bevel up until the wedges are locked in the scleral incision. (Copyright Moorfields Eye Hospital and Keith Barton; reproduced with permission)
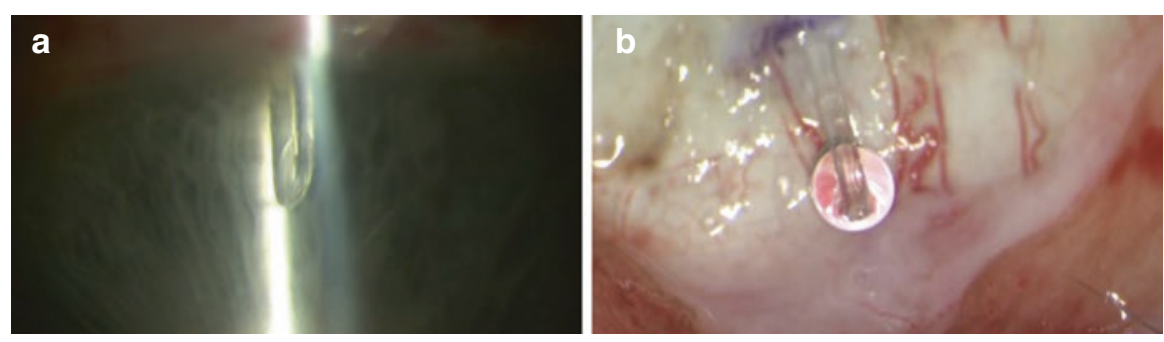

Fig. 7.7 The PRESERFLO ${ }^{\circledR}$ MicroShunt drains aqueous from the anterior chamber to the subconjunctival/subtenon space. (a) The proximal tip of the MicroShunt positioned in the anterior chamber. (b) Egress of aqueous from the distal end of the MicroShunt. (Copyright Moorfields Eye Hospital and Keith Barton; reproduced with permission) 


\subsection{Complications}

\subsubsection{Intraoperative Complications}

Intraoperative complications are uncommon with the PRESERFLO MicroShunt. Potential complications include hyphaema or malposition of the shunt with occlusion by iris or placement of the tip of the device close to the endothelium with risk of endothelial cell damage. These are theoretical risks with any glaucoma drainage device inserted into the anterior chamber and can be avoided with careful surgical technique. Observation of the device directly through the cornea or with the aid of a goniolens can confirm safe placement of the device and in the event the surgeon is not pleased with the placement, the device can simply be pulled out of the scleral tunnel and repositioned in a new tunnel. Confirmation of flow through the device is indicative of no periannular leakage as well as non-obstruction of the tube lumen by iris or cornea.

\subsubsection{Early Postoperative Complications}

Extreme IOP fluctuations within the first week post-operatively with the MicroShunt are rare. Conventional drainage devices such as the Molteno or Baerveldt valves require tying off of the tube lumen to prevent hypotony as well as fenestration of the tube to allow some flow of aqueous humour to maintain the bleb. Similarly, trabeculectomy requires suture tension on the scleral flap to prevent immediate hypotony. These subjective procedures provide a myriad of pressure excursions within the first week post-operatively. The MicroShunt is a fixed flow resistor and the pressure excursions are reduced to a standard deviation of $\pm 4-5 \mathrm{mmHg}$ which can be half that of the aforementioned filtering devices.

Potential early post-operative complications include hyphaema (Fig. 7.8), obstruction of the tube (Fig. 7.9), wound leak and hypotony. Transient numerical hypotony is the most common complication and can be managed with observation. If there is shallowing of the anterior chamber or large choroidal effusions,

Fig. 7.8 Hyphaema associated with the PRESERFLO $^{\circledR}$ MicroShunt. (Copyright Chelvin Sng, FRCSEd; reproduced with permission)

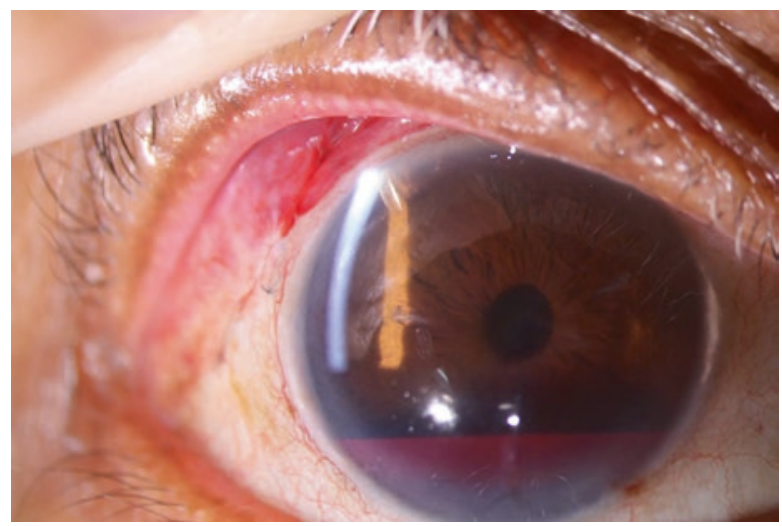


Fig. 7.9 Obstruction of the PRESERFLO ${ }^{\circledR}$

MicroShunt with (a) a blood clot and (b) iris. (Copyright Chelvin Sng, FRCSEd; reproduced with permission)
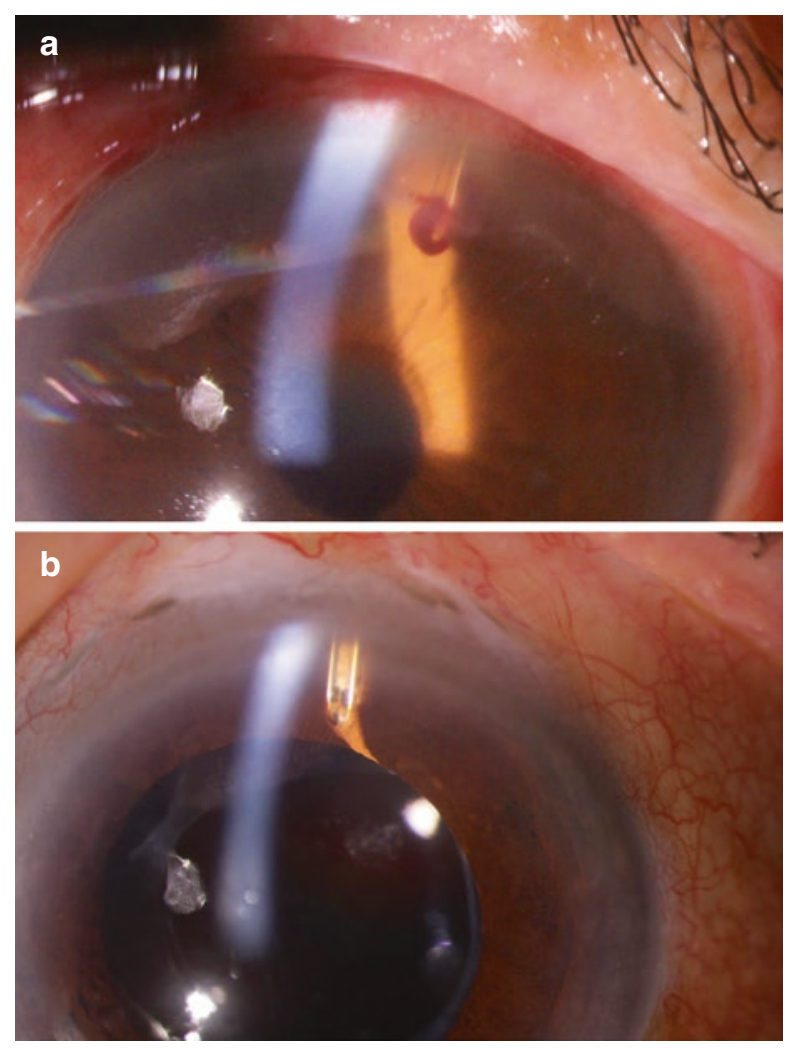

cycloplegics can be commenced and if necessary, injection of viscoelastic into the anterior chamber can be performed. The patient should be observed closely for intraocular pressure spikes if viscoelastic is injected into the anterior chamber. Tube obstruction from iris is a concern if the internal end of the Microshunt is posteriorly located and close to the iris (Fig. 7.9b), and YAG laser or a needle can be used to remove iris from the tip if required. Obstruction of the tube with fibrin or inflammatory debris is usually transient and self-resolving with topical steroids. Wound leaks may be present if conjunctival closure was inadequate. Leaks will usually settle with conservative management, such as bandage contact lenses for anterior leaks. Aqueous misdirection is rare with this device. Similarly, decompression retinopathy has not been observed but is a theoretical concern if there is hypotony.

\subsubsection{Late Complications}

As with trabeculectomy, bleb encystment can occur and may require needling and/ or bleb revision. Both needling and bleb revision are typically performed with antimetabolites to modulate would healing. Erosion or migration of the shunt is 
fortunately rare and can be managed with revision of the shunt and bleb. Blebitis and endophthalmitis have not been reported following MicroShunt implantation but are potential complications of any glaucoma filtration procedure which creates a bleb. Persistent hypotony on two consecutive visits beyond 90 days has not been reported. Lastly, corneal decompensation is a potential complication but this risk can be reduced by preventing the shunt or MMC from coming into contact with the endothelium.

\subsection{Conclusion}

The PRESERFLO MicroShunt is a promising new device that offers substantial reductions in IOP, rivalling those seen with trabeculectomy, in a less invasive and safer procedure. Its improved safety profile may permit earlier surgical intervention in glaucoma management.

\section{References}

1. Investigators A. 7. The relationship between control of intraocular pressure and visual field deterioration. The Advanced Glaucoma Intervention Study (AGIS). Am J Ophthalmol. 2000;130:429-40.

2. Anderson D, Drance S, Schulzer M. Comparison of glaucomatous progression between untreated patients with normal-tension glaucoma and patients with therapeutically reduced intraocular pressures. Am J Ophthalmol. 1998;126:487-97.

3. Chauhan BC, Drance SM. The relationship between intraocular pressure and visual field progression in glaucoma. Graefes Arch Clin Exp Ophthalmol. 1992;230:521-6.

4. Susanna R, De Moraes CG, Cioffi GA, Ritch R. Why do people (still) go blind from glaucoma? Transl Vis Sci Technol. 2015;4:1.

5. Cairns JE. Trabeculectomy. Preliminary report of a new method. Am J Ophthalmol. 1968;66:673-9.

6. Gedde SJ, Herndon LW, Brandt JD, Budenz DL, Feuer WJ, Schiffman JC. Postoperative complications in the Tube Versus Trabeculectomy (TVT) study during five years of follow-up. Am J Ophthalmol. 2012;153:804-14.e1.

7. Gerente VM, Regatieri CVS, Teixeira SH, Paranhos A Jr. Trabeculectomy learning curve: limbus versus fornix based conjunctival flaps-efficacy and complications. Invest Ophthalmol Vis Sci. 2007;48:844.

8. King AJ, Rotchford AP, Alwitry A, Moodie J. Frequency of bleb manipulations after trabeculectomy surgery. Br J Ophthalmol. 2007;91:873-7.

9. King A, Azuara-Blanco A, Tuulonen A. Glaucoma. BMJ. 2013;346:f3518.

10. Arrieta EA, Aly M, Parrish R, et al. Clinicopathologic correlations of poly-(styrene-bisobutylene-b-styrene) glaucoma drainage devices of different internal diameters in rabbits. Ophthalmic Surg Lasers Imaging Retina. 2011;42:338-45.

11. Pinchuk L, Riss I, Batlle JF, et al. The development of a micro-shunt made from poly (styreneblock-isobutylene-block-styrene) to treat glaucoma. J Biomed Mater Res B Appl Biomater. 2017;105:211-21.

12. Lloyd MA, Baerveldt G, Nguyen QH, Minckler DS. Long-term histologic studies of the Baerveldt implant in a rabbit model. J Glaucoma. 1996;5:334-9.

13. Minckler DS, Shammas A, Wilcox M, Ogden T. Experimental studies of aqueous filtration using the Molteno implant. Trans Am Ophthalmol Soc. 1987;85:368. 
14. Stokes K, Coury A, Urbanski P. Autooxidative degradation of implanted polyether polyurethane devices. J Biomater Appl. 1986;1:411-48.

15. Zhao Q, Topham N, Anderson J, Hiltner A, Lodoen G, Payet C. Foreign-body giant cells and polyurethane biostability: in vivo correlation of cell adhesion and surface cracking. J Biomed Mater Res A. 1991;25:177-83.

16. Pinchuk L, Riss I, Batlle JF, et al. The use of poly (styrene-block-isobutylene-block-styrene) as a microshunt to treat glaucoma. Regen Biomater. 2016;3:137-42.

17. Strickler F, Richard R, McFadden S, et al. In vivo and in vitro characterization of poly (styreneb-isobutylene-b-styrene) copolymer stent coatings for biostability, vascular compatibility and mechanical integrity. J Biomed Mater Res A. 2010;92:773-82.

18. Pinchuk L. Biostable elastomeric polymers having quaternary carbons. Google Patents; 1998.

19. Pinchuk L, Wilson GJ, Barry JJ, Schoephoerster RT, Parel J-M, Kennedy JP. Medical applications of poly (styrene-block-isobutylene-block-styrene)(“SIBS"). Biomaterials. 2008;29:448-60.

20. Acosta A, Fernandez V, Lamar P, et al. Ocular biocompatibility of quatromer (polystyrenepolystyrene triblock polymers) for glaucoma applications. Invest Ophthalmol Vis Sci. 2004;45:E-abstract 2929.

21. Acosta AC, Espana EM, Yamamoto H, et al. A newly designed glaucoma drainage implant made of poly (styrene-b-isobutylene-b-styrene): biocompatibility and function in normal rabbit eyes. Arch Ophthalmol. 2006;124:1742-9.

22. Beckers H, Kujovic-Aleksov S, Webers C, Riss I, Batlle J, Parel J-M. One-year results of a three-site study of the MicroShunt (R). Acta Ophthalmol. 2017;95:28-9.

23. Riss I, Batlle J, Pinchuk L, Kato YP, Weber BA, Parel JM. One-year results on the safety and efficacy of the InnFocus MicroShunt depending on placement and concentration of mitomycin C. J Fr Ophtalmol. 2015;38:855-60.

24. Batlle JF, Fantes F, Riss I, et al. Three-year follow-up of a novel aqueous humor microshunt. J Glaucoma. 2016;25:e58-65.

25. Santen Pharmaceutical. Santen Announces Topline Data for DE-128 (MicroShunt) Demonstrating Reductions in IOP and Medication Use in Patients with Glaucoma. Press Release, 30 Aug 2019. https://eyewire.news/articles/santen-announces-topline-data-for-de128-microshunt-demonstrating-reductions-in-iop-and-medication-use-in-patients-with-glaucoma/. Accessed 18 Sep 2019.

Open Access This chapter is licensed under the terms of the Creative Commons Attribution 4.0 International License (http://creativecommons.org/licenses/by/4.0/), which permits use, sharing, adaptation, distribution and reproduction in any medium or format, as long as you give appropriate credit to the original author(s) and the source, provide a link to the Creative Commons license and indicate if changes were made.

The images or other third party material in this chapter are included in the chapter's Creative Commons license, unless indicated otherwise in a credit line to the material. If material is not included in the chapter's Creative Commons license and your intended use is not permitted by statutory regulation or exceeds the permitted use, you will need to obtain permission directly from the copyright holder.

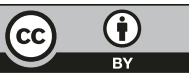

